\title{
S/NB-EVENTS OF JOVIAN DECAMETRIC EMISSION
}

\author{
O. V. Arkhypov*i, and H. O. Rucker*
}

\begin{abstract}
Narrow-band (NB) events in dynamic spectra and their relation with short (S-) bursts are an unresolved enigma of the Jovian decametric emission. This paper is focused on the S/NB-structure with timescales between $0.03 \mathrm{~s}$ and $0.3 \mathrm{~s}$. We show that the main S/NB-phenomenology can be reduced to three main ingredients which are: the dispersion delay of the radio emission, the motion of emitting electrons in the parallel electric field of the standing Alfvén wave, and the shadow effect.
\end{abstract}

\section{Introduction}

Narrow band (NB) event of Jovian decametric emission (DAM) appears in the dynamic spectrum as a stripe of radiation with the frequency band $\Delta f \sim 0.1 \mathrm{MHz}$ oscillating near a certain frequency $f_{o} \gg \Delta f$ (Figure 1). Such an NB-stripe oscillates in the frequency $f$ as well as in the time $t$ forming distinctive hooks (Figure 1b) with an amplitude of $\sim 2 \mathrm{MHz}$ and cycle of $\sim 0.03 \mathrm{~s}$ to $0.3 \mathrm{~s}$. There is a connection between an NB-event and short (S-) bursts (Figure 1c) which is the narrow-band emission, too, but with the fast frequency drift of $\sim-20 \mathrm{MHz} / \mathrm{s}$.

To study the highly complex structure of Jovian S/NB-storms in dynamic spectra, the linear correlation coefficient $r\left(f, f_{o}, \Delta t\right)$ between the spectral intensity in the current $(f)$ and the reference $\left(f_{o}\right)$ frequency channels was calculated with $\Delta t$ time shift for a whole spectrum. We found the hidden regularity in random S-bursts as the double correlation pattern in the form of correlated and anti-correlated stripes in the $f-\Delta t$ plot (Figure 1d) [Arkhypov and Rucker, 2009]). The positive correlation is a trivial result from the concentration of emission in S/NB-features of preferred form and direction. However, the negative correlation reflects the tendency of emission to avoid certain regions in the dynamic spectrum (shadows).

\footnotetext{
* Institute of Radio Astronomy, Ukrainian Academy of Sciences, Chervonopraporna 4, 61002, Kharkiv, Ukraine

† Department of Space Radio Physics, Kharkiv V. N. Karazin National University, Svoboda Square 4, 61077 Kharkiv, Ukraine

‡ Space Research Institute, Austrian Academy of Sciences, Schmiedlstrasse 6, A-8042 Graz, Austria
} 

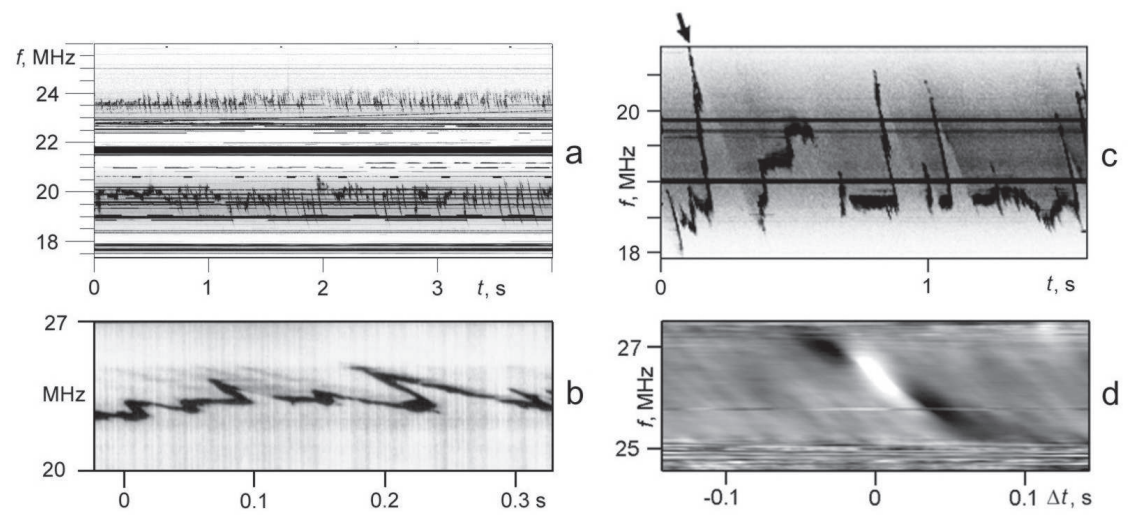

Figure 1: Patterns of Jovian decametric S/NB-emissions: a) two bands of the emission [Arkhypov and Rucker, 2009]; b) NB-hooks [Riihimaa, 1992]; c) the S-bursts and NB-events form clear shadows (whitish) in the diffuse background emission (grey) in analogy to barriers in a flow along the arrowed direction (called the 'modulator' below; [Arkhypov and Rucker, 2011]); d) the typical $2 D$-correlation pattern of the S-bursts reveals the shadows as the dark spots of anti-correlation [Arkhypov and Rucker, 2009].

There are enigmatic associations of DAM at certain radio frequencies. Short S-bursts and narrow band events of DAM have a tendency to be captured at certain frequencies in dynamic spectra (Figure 1a-c). This behavior is described as an event of type $\mathrm{N}$ [Riihimaa, 1985].

In this paper we describe the simple phenomenological model reproducing the aforesaid properties of S/NB emissions.

\section{Motion of an Emitter}

It is believed that the S-burst drift in a dynamic spectrum reflects the motion of a radio source (a bunch of electrons) along a magnetic field upwards with a decrease of the emission frequency in accordance with the local gyrofrequency of electrons [e.g., Hess et al., 2007a]. In our model, this motion is controlled by a standing Alfvén wave, which is predicted in the ionosphere Alfvén resonator of Jupiter [Su et al., 2006].

The parallel electric field $E_{1}$, generated by an Alfvén wave, is proportional to the perpendicular magnetic disturbance $\delta B$ (e.g., Eq. 13 in Hess et al. [2007b]). This wave disturbance is assumed in the simplest, harmonic form:

$$
E_{1}=K \delta B=E_{o} \sin \left[\frac{2 \pi t}{P}-\frac{2 \pi\left(X-X_{o}\right)}{\lambda}\right]
$$

where $K$ is the factor of proportionality; $E_{o}=K \delta B_{o}$ is the amplitude of the electric field corresponding to the magnetic amplitude $\delta B_{o} ; P$ is the time period of the wave; $\lambda$ is the 
wavelength; $X$ is the spatial coordinate along the magnetic field line; $X_{o}$ is the coordinate of the reference node of the Alfvén wave.

As the Alfvén wave is reflected from the Jovian ionosphere, there is another wave propagating in the opposite direction: $E_{2}=E_{o} \sin \left[(2 \pi / P) t+2 \pi\left(X-X_{o}\right) / \lambda\right]$. The superposition of the parallel electric fields of these waves forms the standing wave as the product of temporal and spatial oscillations:

$$
E=E_{1}+E_{2}=2 E_{o} \sin \left(\frac{2 \pi t}{P}\right) \cos \left[\frac{2 \pi\left(X-X_{o}\right)}{\lambda}\right],
$$

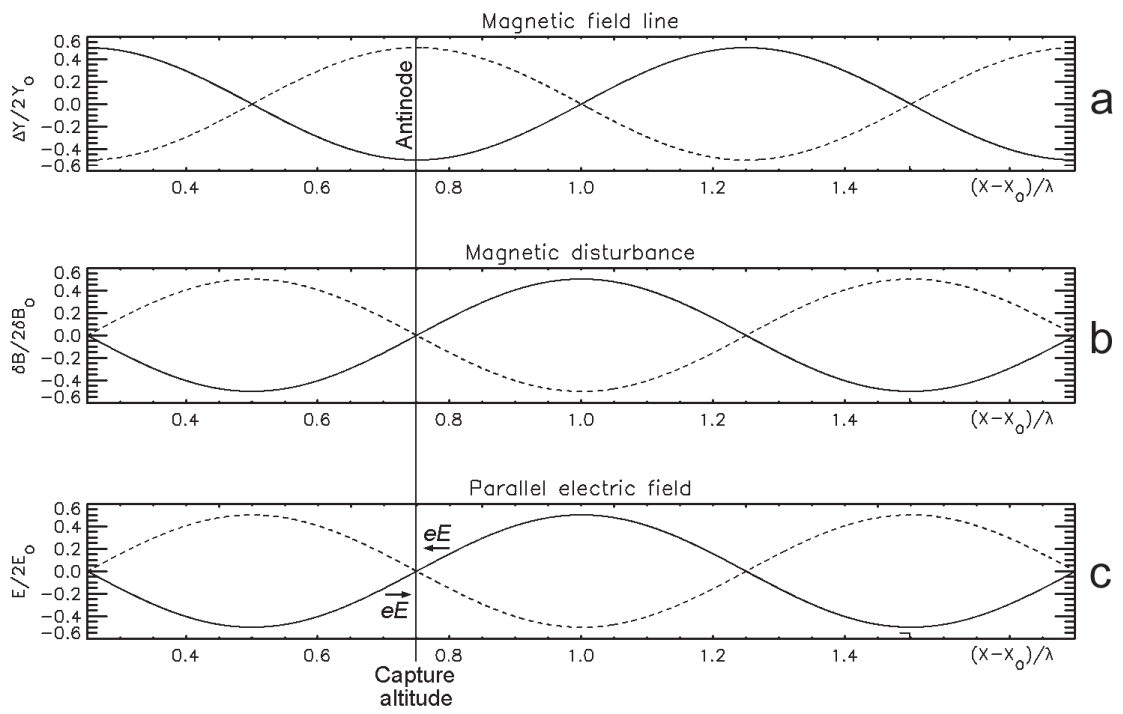

Figure 2: Idealized variations in the standing Alfvén wave with the coordinate $X$ along the magnetic field line: a) the perpendicular deflection $\Delta Y$ from undisturbed magnetic field line; $b$ ) the corresponding pulsation of perpendicular component of the magnetic field $\delta B ; c)$ the parallel electric field E according to Eq. 1 and 2. The solid and dashed curves are calculated for the maximal deformations with the amplitudes of $Y_{o}, \delta B_{o}, E_{o}$, but with the time difference of $P / 2$. The converging Coulomb force at the $E$ sign inversion ( $E E$ arrows) could entrap electrons at the wave antinode.

Formally, such standing Alfvén wave can capture electrons. It is possible because $E$ inverts its sign in space. Correspondingly, the converging Coulomb force can entrap electrons near the wave antinodes (Fig. 2).

The parallel (to the magnetic field) typical velocity of electrons $V$ in the plasma is proportional to the electric field according to the Ohm's law in resistive approximation: $E=\eta J$, where $\eta$ is is the plasma resistivity; $J=e n V$ is the electric current density; $e$ is the electric charge of the electron; $n$ is the electron number density. As the DAM is generated 
by cyclotron maser instability in the inhomogeneous Jovian magnetic field, there is certain relation in $E$ (Eq. 2) between the spatial coordinate $X$ and the DAM's frequency $f$ near the local gyrofrequency of electrons. In linear approximation at short distances, the substitutions of the linear dimension of distance $X$ to frequency $(f)$ and $X_{o} \rightarrow f_{o}$ are acceptable. Hence, the equation of motion along the magnetic field line is:

$$
V=\frac{2 E_{o}}{\eta e n} \sin \left(\frac{2 \pi t}{P}\right) \cos \left[\frac{2 \pi\left(f-f_{o}\right)}{\lambda_{f}}\right],
$$

where $\lambda_{f}=\left(d f_{c e} / d X\right) \lambda$ is the wavelength in frequency scale. To study the possible connection between the electron captures and associations of Jovian narrowband emissions with certain frequencies, we should compare the solutions of Eq. 3 and dynamic spectra of DAM.

The emitter trajectory $f(t)$ in the dynamic spectrum of DAM is found with numerical integration:

$$
\begin{gathered}
f_{i+1}=f_{i}+V\left(t_{i}, f_{i}\right) \Delta t, \\
t_{i+1}=t_{i}+\Delta t+\delta t_{i},
\end{gathered}
$$

where $\Delta t$ is the step in time; $V=\left[V\left(t_{i}\right)+V\left(t_{i}+1\right)\right] / 2$ is the frequency drift rate, and $\delta t_{i}$ is the time delay due to the DAM propagation. The frequency drift rate could be found from Eq. (3):

$$
V\left(t_{i}, f_{i}\right) \equiv d f / d t=A_{v} \sin \left(\frac{2 \pi t_{i}}{P}\right) \cos \left[\frac{2 \pi\left(f_{i}-f_{o}\right)}{\lambda_{f}}\right],
$$

where $A_{v}=\frac{2 E_{o}}{\eta e n}$ is the amplitude of the drift rate.

For more realistic simulations, the noise $\delta V\left(t_{i}\right)$ and the random jumps in the phase $\varphi$ is added in Eq. 6:

$$
V\left(t_{i}, f_{i}\right)=A_{v} \sin \left(\frac{2 \pi t_{i}}{P}+\varphi\right) \cos \left[\frac{2 \pi\left(f_{i}-f_{o}\right)}{\lambda_{f}}\right]+\delta V\left(t_{i}\right),
$$

where $\delta V\left(t_{i}\right)=\sum_{k=1}^{N=50} \delta v_{k} \cos \left[\left(2 \pi / p_{k}\right) t_{i}+\varphi_{k}\right]$ is the arbitrary choice sum of harmonics with the random amplitudes $\delta v_{k}$, the random period $p_{k}$ in the interval from 0.03 to 0.3 $\mathrm{s}$, and the random phase $\varphi_{k}$. The phase $\varphi$ is constant during the period $P$, but $\varphi$ jumps to another random value for the next cycle. The physical interpretation of such phase jumps is the excitation of an ionosphere/magnetosphere resonator with accidental wave packets. In that case the phase of a standing wave in the resonator is variable. However, the resonator's borders and, hence, the wave antinodes are fixed.

Equations 4, 5 and 7 are used in our calculation of the trajectories of emitting electrons in the DAM dynamic spectrum.

\section{Dispersion Delay}

Let there be a dispersion delay of the radio emission. This assumption has been proposed before [Willes, 2002]. Moreover, there is an obvious time-delay effect in NB-events: 
NB-hooks have been observed, where the emission from one narrow-band is observed simultaneously at 2 to 3 different frequencies (Fig. 1c, e.g., at 0.22 s). Apparently, the NB-oscillation in the spectrum (e.g., Fig. 1a) is systematically distorted into an asymmetric, saw-tooth form. Therefore, the dispersion delay seems to be an important ingredient for models of NB-events at least.

The dispersion delay in Eq. 5 is modeled in the simplest, linear form

$$
\delta t_{i}=\frac{f_{i}-f_{o}}{D_{d}}
$$

where $D_{d}$ is the frequency drift rate from the dispersion (usually $-20 \mathrm{MHz} / \mathrm{s}$ ).

\section{Shadow Effect}

As a result of the previous analysis, the concept of the modulator has been proposed [Arkhypov and Rucker, 2011]. The modulator is an activating or amplifying agent, which drifts in the dynamic spectrum toward lower frequencies to stimulate the generation process in the radio source, i.e., the band of emission in the dynamic spectrum (Figure 1d). After the source interaction, the modulator is shielded; one can not stimulate the emission afterwards. Hence, the emission band makes the shadow in a certain region of the spectrum which is observed sometimes as 'shadow event'(Figure 1d). This 'shadow effect' regularizes the random NB-oscillations into realistic structures in the synthetic spectrum.

The shadow effect is absent, if the dispersion drift rate $D_{d}$ is equal to the frequency drift rate of the modulator $\left(D_{m}\right)$. If $\left|D_{d}\right|>\left|D_{m}\right|$, the NB-source can be in its shadow, where the modulator has been shielded by the same source before. This auto-shadowing is present in certain regions of the dynamic spectrum. Let the frequency of the radio emission is the function of time $\Phi(t)$ in the radio source. Then the shadow begins at the point with the coordinates $f_{*}=\Phi(t)$ and $t_{*}=t+\delta t$ (time $t$ corrected for the dispersion delay from Eq. 8). Then the shadow extends along the modulator drift line $f=f_{*}+D_{m}\left(t-t_{*}\right)$. The set of these lines forms the spacious shadow.

Although the modulator concept correlates with the model of an S-burst source as an interaction region between the upward flux of electrons and the radio wave generated [Willes, 2002], it is difficult to interpret this shadow principle in terms of a concrete physical model without any in situ measurements. However, the NB auto-shadowing reproduces the various features in the dynamic spectra in form of S-burst groups and sinuous NB with the positive frequency drift, the flag-like features, zigzag bursts and the periodical points and streaks [Arkhypov and Rucker, 2011]. Therefore, the shadow effect seems to be an important ingredient of the DAM theory.

\section{Synthetic and Real Spectra}

The parameter $D_{d}$ is adopted mainly $-20 \mathrm{MHz} / \mathrm{s}$ in accordance with the average drift rate of S-bursts (from -15 to $-25 \mathrm{MHz} / \mathrm{s}$ at frequencies $15<f<35 \mathrm{MHz}$; Fig. 3b in [Hess et 
al., 2007a]). The standing and propagating Alfvén wave periods $\left(P\right.$ and $\left.p_{k}\right)$ are selected in accordance with other authors (0.05 s, [Su et al., 2006]; $0.2 \mathrm{~s}$, [Hess et al., 2007b]). The Alfvén wavelength $\left(\lambda_{f}\right)$ is chosen arbitrarily but in accordance with the predicted size of the ionospheric Alfvén resonator $\left(\sim 0.1 R_{J}\right.$; [Su et al., 2006]) and the frequency interval between bands of S-bursts (2.5 MHz to $4 \mathrm{MHz}$; [Arkhypov and Rucker, 2009]). We don't optimize the model parameters to achieve the "best fit quality". The parameters are chosen approximately for demonstration only qualitative similarity with the experimental spectra.

Figure 3 shows the results of our numerical integration of Equations 4, 5 and 7 with $\delta V\left(t_{i}\right)=0$ and $\varphi=0$. The motion of the emitter at a distance from antinodes of the Alfvén wave appears as the inclined oscillations in the dynamic spectrum (Fig. 3c). These features resemble the wave-like NB-events (Fig. 3a). However, the clipped form appears (Fig. 3d) if antinodes are at the borders of the motion range. In this case the flat parts of the curve depict the capture of the electron at the anti-node of the standing Alfvén wave, where $E$ inverts its sign and captures the particle (Fig. 2). Resembling emissions at $f=$ const are typical for NB-events of DAM [Riihimaa, 1991].
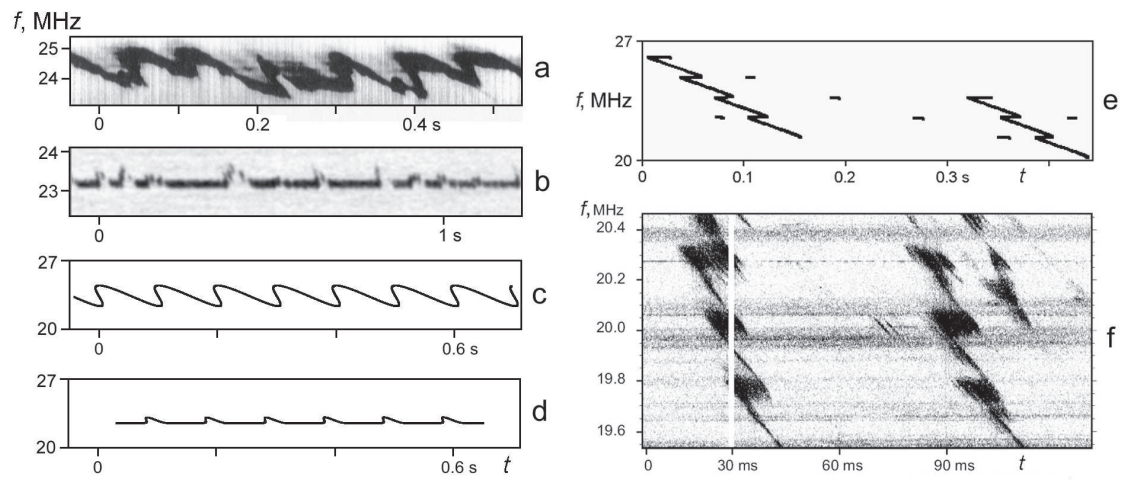

Figure 3: Resemblance between real spectra of S/NB-events (a)[Riihimaa, 1992]; b) [Riihimaa, 1991]; f) [Litvinenko et al., 2009]) and the calculated spectra: c) the hooks modeled with $\delta V\left(t_{i}\right)=\varphi=0$ at the Alfvén wave node $\left(D_{d}=-40 \mathrm{MHz} / \mathrm{s} ; D_{m}=-30 \mathrm{MHz} / \mathrm{s} ; P=0.1 \mathrm{~s}\right.$; $\left.A_{v}=73 \mathrm{MHz} / \mathrm{s}\right) ; d$ ) the emitter captions at the antinode; e) the step-like captures at same frequencies are a result of noise addition $\delta V$ in Eq. $7\left(D_{d}=-20 \mathrm{MHz} / \mathrm{s} ; D_{m}=-15 \mathrm{MHz} / \mathrm{s}\right.$; $\left.P=0.05 \mathrm{~s} ; \lambda_{f}=2.3 \mathrm{MHz} ; A_{v}=879 \mathrm{MHz} / \mathrm{s} ; 0.44 \leq \delta v_{k} \leq 4.40 \mathrm{MHz} / \mathrm{s}\right)$.

The noise $\delta V\left(t_{i}\right) \neq 0$ disturbes the trajectory permitting the electron's transfer from one node region to another. If the amplitude of NB-oscillation is much longer than $\lambda_{f} / 2$, the electron is captured at successive antinodes, and the step-like trajectory is formed in the dynamic spectrum. The shadow modulation (Section 4) retains only observed zig-zag forms in the dynamic spectrum (Fig. 3e).

If the random jumps in $\varphi$ are added in Eq. 7 with $\delta V\left(t_{i}\right) \neq 0$, the realistic S-burst train transforming into NB emission is a result of the integration in combination with the shadow effect (Fig. 4a). 

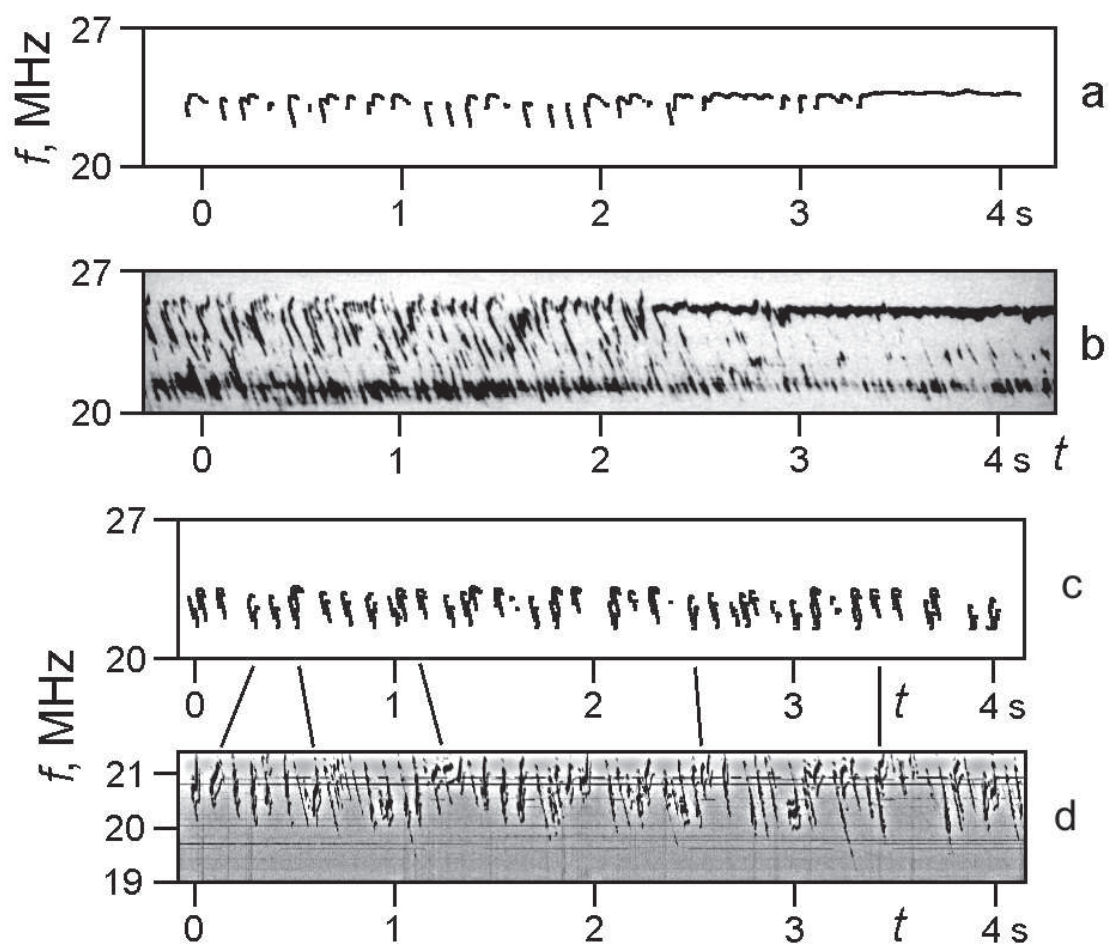

Figure 4: Effect of the random jumps in $\varphi$ plus the noise $\delta V\left(t_{i}\right) \neq 0$ : a) the transforming of $S$ band-like oscillations into the NB-like capture (starting at $t=3.3 \mathrm{~s}$ ) resembles the real dynamic spectrum in (b)(Fig. 11 in [Riihimaa, 1991]; c) the close disposition of the independent curves which are calculated as in (a) resembles the real spectrum with double $S$-bursts (2002 August 9, 11:30 UT; UTR-2 radio telescope was used within the frame of the INTAS project 03-51-5727). The parameter sets are: in Fig. 4a) $D_{d}=-20 \mathrm{MHz} / \mathrm{s}, D_{m}=-10 \mathrm{MHz} / \mathrm{s} ; P=0.1 \mathrm{~s} ; \lambda_{f}=4.7$ $\mathrm{MHz} ; A_{v}=44 \mathrm{MHz} / \mathrm{s} ; 0.044 \leq \delta v_{k} \leq 0.44 \mathrm{MHz} / \mathrm{s} ;$ in Fig. 4c) $D_{d}=-20 \mathrm{MHz} / \mathrm{s} ; D_{m}=-10$ $\mathrm{MHz} / \mathrm{s} ; P=0.1 \mathrm{~s} ; \lambda_{f}=4.7 \mathrm{MHz} ; A_{v}=44 \mathrm{MHz} / \mathrm{s}, 0.044 \leq \delta v_{k} \leq 0.44 \mathrm{MHz} / \mathrm{s}$.

The superposition of two independent curves in close proximity (Fig. 4c) resembles the real splitting structures in DAM spectra in Fig. 4d.

\section{Conclusions}

1. Resemblance between the calculated curves and real DAM spectra supports our model which is based on the shadow effect, dispersion delay of the emission, and the motion of the emitter by the standing Alfvén wave. Hence, these components are important ingredients of any future developed DAM theory. 
2. Practically, the characteristic dash-line appearance of $\mathrm{S} / \mathrm{NB}$ radiation in the dynamic spectrum could be considered as a result of superposition of numerous shadows. The decreasing amplitude of the emitter oscillations can transform S-band into NB-event like in Figure 4a.

3. Antinodes of the standing Alfvén wave could capture the DAM radio-sources forming the bands of S-bursts, NB-emissions and zig-zag forms of S-bursts in the dynamic spectrum.

4. The splitting S-bursts suggest several independent radio-sources (in different magnetic flux tubes?) oscillating in the parallel electric field of the same standing Alfvén wave.

\section{References}

Arkhypov, O.V. and H. O. Rucker, Regularity in dynamic spectra of Jovian radio decameter S-bursts, Astron. Astrophys., 496, 269-274, 2009.

Arkhypov, O. V. and H. O. Rucker, Shadows in S/NB-events of jovian decametric emission, Icarus, 211, 603-608, 2011.

Hess, S., P. Zarka, and F. Mottez, Io-Jupiter interaction, millisecond bursts and fieldaligned potentials, Planet. Space Sci., 55, 89-99, 2007a.

Hess S., F. Mottez, P. Zarka, Jovian S-bursts generation by Alfvén waves, J. Geophys. Res., 112, A11212, 2007b.

Litvinenko, G. V., A. Lecacheux, H. O. Rucker, A. A. Konovalenko, B. P. Ryabov, U. Taubenschuss, V. V. Vinogradov, V. E. Shaposhnikov, Modulation structures in the dynamic spectra of Jovian radio emission obtained with high time-frequency resolution, Astron. Astrophys., 493, 651-660, 2009.

Riihimaa, J. J., Bursts of type N in Jupiter's decametric radio spectra, Earth Moon Planets, 32, 9-19, 1985.

Riihimaa, J. J., Evolution of the spectral fine structure of Jupiter's decametric S-storms, Earth Moon Planets, 53, 157-182, 1991.

Riihimaa, J. J., Wide-range high-resolution S-burst spectra of Jupiter, Univ. of Oulu, Oulu, 1992.

Su, Y.-J., S. T. Jones, R. E. Ergun, F. Bagenal, S. E. Parker, P. A. Delamere, R. L. Lysak, Io-Jupiter interaction: Alfvén wave propagation and ionospheric Alfvén resonator, J. Geophys. Res., 111, A6, doi: 10.1029/2005JA011252, 2006.

Willes, A. J., Jovian S burst drift rates and $\mathrm{S}$ burst/L burst interactions in a phasebunching model, J. Geophys. Res., 107, A5, SMP 7-1, doi:10.1029/2001JA000282, 2002 . 